\title{
Análise de Redes Sociais e de Complexidade: Uma Estratégia para Aprimoramento de Sistemas Éticos para a Ciência Aberta?
}

\author{
Social Network Analysis and Complexity Analysis: A Strategy for \\ Enhancing Ethical Systems for Open Science?

\section{Análisis de Redes Sociales y de Complejidad: Una Estrategia para Mejorar los Sistemas Éticos para la Ciencia Abierta?}

\author{
Jorge Henrique Cabral Fernandes|jhcf@unb.br \\ Universidade de Brasília, Departamento de Ciência da Computação. Brasília, DF, Brasil. \\ Wagner de Jesus Martins | wagner.martins@fiocruz.br \\ Fundação Oswaldo Cruz, Gerência Regional de Brasília, Brasilia, DF, Brasil. \\ Ricardo Barros Sampaio | ricardo.sampaio@fiocruz.br \\ Fundação Oswaldo Cruz, Gerência Regional de Brasília, Brasilia, DF, Brasil.
}

\section{Resumo}

As expressões de conhecimento aberto, englobadas em seu maior nível pela ciência aberta (open science), pressupõem abertura das barreiras aos relacionamentos estabelecidos entre as partes que desejam produzir conhecimento, desde que solucionados aspectos da ética e da confiança, com o objetivo de promover a cidadania. Ética, confiança e cidadania são questões complexas e multifacetadas, cujas respostas são tratadas de forma diferente em diferentes domínios do conhecimento, tais como em saúde e em ecologia. Na ecologia a construção da ciência aberta tem se mostrado muito bem sucedida, enquanto que no campo da saúde ainda são dados os passos iniciais. Esse artigo explora as possíveis diferenças no tratamento da primeira dessas questões, a ética, comparando-a nas ciências da ecologia e da saúde. O debate abre espaço para postulados sobre as possibilidades e barreiras à construção da ciência aberta em saúde, por meio de estudos de análise de redes sociais e de complexidade.

Palavras-chave Complexidade; Análise de Redes Sociais; Ética; Ciência Aberta. 


\begin{abstract}
The expressions of open knowledge, embraced at their highest level by open science, presuppose the opening of barriers to established relationships between the parties wishing to produce knowledge, provided that aspects of ethics and trust are solved, with the objective of promoting citizenship. Ethics, trust and citizenship are complex and multifaceted issues, whose responses are treated differently in different domains of knowledge, such as health and ecology. In ecology the construction of open science has been very successful, while in the field of health the initial steps are still being taken. This article explores the possible differences in the treatment of the first of these issues, ethics, comparing it in the sciences of ecology and health. The debate opens space for postulates about the possibilities and barriers to the construction of open health science, through studies of social network analysis and complexity.
\end{abstract}

Keywords: Complexity; Social Network Analysis; Ethics; Open Science; Bioethics.

\title{
Resumen
}

Las expresiones de conocimiento abierto, englobadas en su mayor nivel por la ciencia abierta (open science), presuponen la apertura de las barreras a las relaciones establecidas entre las partes que desean producir conocimiento, siempre que se solucionen aspectos de la ética y la confianza, con el objetivo de promover la ciudadanía. La ética, la confianza y la ciudadanía son cuestiones complejas y multifacéticas, cuyas respuestas se tratan de forma diferente en diferentes ámbitos del conocimiento, tales como en salud y en ecología. En la ecología la construcción de la ciencia abierta se ha mostrado muy exitosa, mientras que en el campo de la salud todavía se dan los pasos iniciales. Este artículo explora las posibles diferencias en el tratamiento de la primera de estas cuestiones, la ética, comparándola en las ciencias de la ecología y la salud. El debate abre espacio para postulados sobre las posibilidades y barreras a la construcción de la ciencia abierta en salud, a través de estudios de análisis de redes sociales y de complejidad.

Palabras clave: Complejidad; Análisis de Redes Sociales; Ética; Ciencia Abierta; Bioética.

\section{Audiência}

Formuladores de políticas públicas em ciência aberta. Investigadores em bioética.

\section{Ética, Confiança e Cidadania na Ciência Aberta}

O conhecimento aberto, englobado pela ciência aberta, pressupõe eliminar barreiras aos relacionamentos entre partes que desejam produzir conhecimento, desde que solucionados aspectos da ética ${ }^{1}$ e confiança ${ }^{2}$, com o objetivo de promover a cidadania ${ }^{3,4}$.

$\mathrm{Na}$ ecologia, a ciência aberta tem se mostrado bem sucedida ${ }^{5,6,7,8}$, a partir do início deste século ${ }^{3}$. Em outros campos do conhecimento, inclusive em saúde, ainda são dados passos iniciais9. Segundo Cooper10, ecologia é, dentre outras ciências da natureza, aquela que mais se aproxima das ciências sociais, dado seu papel no desenho e implementação de políticas ambientais. Seriam os desafios éticos, de confiança e cidadania, que possibilitaram a abertura da ciência na ecologia, de superação equivalente no campo da saúde? Existiriam formas de transposição da ética em ecologia para conduzir à pesquisa aberta em saúde?

\section{Ética em Saúde e Ecologia}

Na saúde, questões éticas são primordialmente abordadas pela bioética, definida como tratamento "das questões morais que surgem a partir do uso de novos procedimentos médicos ou dos avanços na biotecnologia" ${ }^{11}$. Alguns desafios da bioética decorrem do mapeamento das relações que o avanço biotecnológico estabelece com os distintos arcabouços históricos, linguísticos, políticos, religiosos, legais e culturais que caracterizam uma humanidade heterogênea nos âmbitos local, regional e mundial ${ }^{11}$. 
Já na ecologia, a referência é a ética ambiental, abordada em três principais correntes de pensamento1: antropocentrismo, biocentrismo e ecocentrismo.

O antropocentrismo, ao focar no ser humano, debate, por exemplo, a utilização de tecnologias diversas que podem impactar saúde e bem-estar de indivíduos e populações ${ }^{1}$ como fim maior de um sistema moral, e assim se aproxima das questões correntemente tratadas pela bioética.

De forma aproximada ao antropocentrismo, o biocentrismo principia questionando a influência de intervenções humanas sobre quaisquer espécimes que sentem dor e prazer. Mesmo com a expansão posterior desses questionamentos, limitações do biocentrismo em apresentar soluções práticas para problemas na relação do ser humano com o meio ambiente ${ }^{1} \mathrm{o}$ tornam pouco relevante.

A ética do ecocentrismo ambiental (holismo ou fisiocentrismo ${ }^{12}$, ou simplesmente ética ecológica ${ }^{13}$ ), deriva do biocentrismo por atribuição de interesse, valor ou significância moral a espécies e ecossistemas ${ }^{1}$, permitindo o desenvolvimento de percepção holística de relações envolvidas.

Dessa forma, abordagens éticas que podem fomenta o atual estado de abertura da ecologia às práticas da ciência aberta se alinham mais ao ecocentrismo que ao antropocentrismo, deslocando o foco de apreciação moral e valorativa das questões ligadas diretamente ao ser humano, possibilitando o desenvolvimento de percepções sistêmicas ${ }^{14,15}$, holísticas ${ }^{13}$ e complexas ${ }^{16}$ de interrelações e valores presentes em elementos fora do domínio humano imediato.

A introdução de uma perspectiva ecocêntrica na bioética poderia se dar com a incorporação analítica de fenômenos investigados nas ciências da complexidade, das redes complexas, da análise de redes sociais e da análise estrutural.

\section{Complexidade e Redes}

O conceito contemporâneo de sistema substitui visões de um mundo fechado pela de um mundo composto por sistemas abertos, ou por redes. Para explicar como surge a organização sistêmica e complexa dos seres vivos naturais ou artificiais ${ }^{17}$, nos últimos 30 anos surgiu um paradigma de estudo denominado ciência da complexidade ${ }^{16,18}$ ou dos sistemas complexos adaptativos ${ }^{19}$. A complexidade fundamenta-se na física, e investiga propriedades dos sistemas interativos complexos, provendo respostas para a emergência de estruturas coerentes e intencionais, a partir das interações de componentes mais simples e que muitas vezes não possuem comportamento intencional. Seria a complexidade uma base para aprimoramento da ética em ciência em saúde, fomentando maior sucesso na abertura da ciência em saúde?

No final do Século XX, a disponibilização de imensos volumes de dados empíricos sobre relacionamentos entre agentes encaminhou análise da complexidade em direção à análise das redes ${ }^{20,21}$. Fenômenos da complexidade, antes apenas evidenciados em sistemas físicos simulados, passaram a ser investigados em registros de ação humana.

\section{Análise de Redes Sociais}

Conquanto estudos sobre redes e sistemas complexos representem uma conjugação de esforços multi e transdisciplinares para o estudo de fenômenos em sistemas de natureza eminentemente física e tecnológica, a análise de redes sociais (ARS) conjuga esforços análogos, na complexidade em sistemas sociais ${ }^{22,23}$.

A ARS propõe adotar o paradigma de análise estrutural ${ }^{24}$, em oposição simultânea às visões holísticasistêmicas e individualistas, ainda proeminentes em vários métodos de pesquisa social.

A análise estrutural supõe serem tendenciosas as formas de se determinar aprioristicamente como surgem grupos e posições sociais, bem como normas que deles derivam. Mais ainda, posições sociais e normas, entre 
outros fenômenos, não surgem por uma relação abstrata, conceitual, mas sim "devido às relações concretas entre indivíduos que, dando forma à estrutura social, facilitam acessos de alguns a recursos.”24.

\section{Por uma Bioética Complexa e em Redes}

Enquanto questões que interligam complexidade e ética em saúde focam na complexidade inerente à prática da ética ${ }^{25}$, ou nas "tensões entre as responsabilidades sociais da ciência e aquelas decorrentes da produção de resultados comercializáveis de uma forma custo-efetiva, pelas modernas tecnologias corporativas"26, a análise estrutural apresenta formas construtivas de abordagem da complexidade, não como uma contingência que dificulta a ação, mas como uma oportunidade para aprimoramento análises e sínteses.

A teoria clássica dos sistemas investiga fenômenos como feedback, controles e homeostase ${ }^{27}$. A complexidade investiga fenômenos como $^{18}$ controle emergente, criticalidade auto-organizada, evolução na borda ordem-caos e fitness landscapes, entre outros. Redes complexas investigam fenômenos como ${ }^{20}$ liberdade de escala (scale-free), estruturas de mundo pequeno (small world), de centro-periferia e de força de laços fracos e fortes. Análise de redes sociais $23,24,28,29$ investiga fenômenos como homofilia, propinquidade, buracos estruturais, grupos coesos, intermediações, capital social, influência e difusão, entre outros.

Ao incorporar de forma incremental o arcabouço conceitual desses fenômenos citados, a bioética poderia ser contemplada com um grande arsenal analítico, talvez capaz de equacionar um conjunto de questionamentos morais que a conduzirão a uma abordagem ecocêntrica, possibilitando a redução de atuais barreiras à construção de uma ciência aberta em saúde.

\section{Referências}

1. Post SG. Encyclopedia of Bioethics. 3rd ed. USA: Macmillan Reference USA; 2004. 1227 p. (D-H; vol. 2).

2. Grand A, Wilkinson C, Bultitude K, Winfield AFT. Open Science: A New "Trust Technology"? Science Communication. 2012 Sep 13;34(5):679-89.

3. Silvertown J. A new dawn for citizen science. Trends in Ecology \& Evolution. 2009 Sep;24(9):467-71.

4. Hochachka WM, Fink D, Hutchinson RA, Sheldon D, Wong W-K, Kelling S. Data-intensive science applied to broad-scale citizen science. Trends in Ecology \& Evolution. 2012 Feb;27(2):130-7.

5. Reichman OJ, Jones MB, Schildhauer MP. Challenges and opportunities of open data in ecology. 2011;331(6018):703-5.

6. Michener WK, Jones MB. Ecoinformatics: supporting ecology as a data-intensive science. Trends in Ecology \& Evolution. 2012 Feb;27(2):85-93.

7. Rocchini $D$, Neteler M. Let the four freedoms paradigm apply to ecology. Trends in Ecology \& Evolution. 2012 Jun;27(6):310-1.

8. Stokstad E. Open-Source ecology takes root across the world. Science. 2011;334:308-9.

9. Munafò MR, Nosek BA, Bishop DVM, Button KS, Chambers CD, Percie du Sert N, et al. A manifesto for reproducible science. Nature Human Behaviour. 2017 Jan 10;1:0021.

10. Cooper GJ. The Science of the Struggle for Existence: On the Foundations of Ecology. UK: Cambridge University Press; 2003. 319 p. (Cambridge Studies in Philosophy and Biology).

11. Lerner KL, Lerner BW. Medicine, Health, and Bioethics: Essential primary sources. USA: Thomson Gale; 2013. $513 \mathrm{p}$.

12. Gorke M. The Death of Our Planet's Species: A Challenge to Ecology and Ethics. USA: Island Press; 2003. $407 \mathrm{p}$. 
13. Kisner W. Ecological Ethics and Living Subjectivity in Hegel's Logic: The Middle Voice of Autopoietic Life. USA: Palgrave Macmillan; 2014. 327 p.

14. Meadows D. Thinking in Systems. EUA: Chelsea Green; 2001.

15. Andrade A, Seleme A, Rodrigues L, Souto R. Pensamento Sistêmico: Caderno de Campo. São Paulo: Bookman; 2006.

16. Cowan GA, Pines D, Meltzer D, editors. Complexity: Metaphors, models and reality. USA: AddisonWesley; 1994. (Santa Fé Institute Studies in the Sciences of Complexity; vol. XIX).

17. Langton CG, editor. Artificial Life: An Overview. USA: MIT Press; 1995.

18. Kauffman S. At Home in the Universe: the Search for Laws of Self-Organization and Complexity. New York: Oxford University; 1996. 312 p.

19. Miller JH, Page SE. Complex Adaptive Systems: An introduction to computational models of social life. USA: Princeton University Press; 2007. 263 p.

20. Newman MEJ. Networks: An Introduction. USA: Oxford University Press; 2010. 720 p.

21. Lissack MR. Complexity: the science, its vocabulary, and its relation to organizations. Emergence: Complexity and Organization. 1999 Jan;1(1):110-26.

22. Wasserman S, Faust K. Social Network Analysis: Methods and applications. USA: Cambridge University Press; 1994. 825 p.

23. Valente TH. Network interventions. Science. 2012;337:49-53.

24. Degenne A, Forse M. Introducing Social Networks. UK: Sage Publications; 1999. 248 p. (ISM Introducing Statistical Methods).

25. Goldim JR. Bioética complexa: uma abordagem abrangente para o processo de tomada de decisão. Revista da AMRIGS. 2009 Jan;53(1):58-63.

26. Strohman RC. The complexity of bioethics. Nat Biotech. 2001 Nov;19(11):1007-1007.

27. Bertalanffy L von. General System Theory: foundation, development, applications (revised edition). New York - USA: George Braziller; 1969. 295 p.

28. Kadushin C. Understanding Social Networks: Theories, concepts, and findings. USA: Oxford University Press; 2012.

29. De Nooy W, Mrvar A, Batagelj V. Exploratory Social Network Analysis with Pajek. USA: Routldge; 2005. 362 p. (Structural Analysis in the Social Sciences). 